\title{
Correct Equations for Minimum Noise Measure of a Microwave Transistor Amplifier
}

\author{
Clive Poole ${ }^{\circledR}$ and Ryan Grammenos, Member, IEEE
}

\begin{abstract}
New equations for circles of constant noise measure in the source reflection coefficient plane are presented, and these are used to derive closed-form expressions for the minimum noise measure $\left(M_{\min }\right)$ and the associated source termination $\left(\Gamma_{\mathrm{om}}\right)$ for a single-stage microwave transistor amplifier. The new equation for $M_{\min }$ replaces an incorrect equation that was previously published by one of the authors. The validity of the new equations has been verified by numerical calculation, simulation, and comparison with results obtained by other authors using different methodologies.
\end{abstract}

Index Terms-Advanced design system (ADS), characteristic noise matrix, low-noise amplifier, metal-semiconductor fieldeffect transistor (MESFET), multistage amplifiers, noise figure, noise measure, $S$-parameters.

\section{INTRODUCTION}

$\mathbf{I}$ N 1985, one of the authors, together with Dr. D. K. Paul, published an article [1] outlining a design methodology for achieving minimum noise measure $(M)$ for a single-stage transistor amplifier, where the quantity $M$ is originally defined by Haus and Adler [2]

$$
M=\frac{F-1}{\left(1-\frac{1}{G_{a}}\right)}
$$

where

$$
\begin{aligned}
F & =\text { noise factor of the stage; } \\
G_{a} & =\text { available power gain of the stage. }
\end{aligned}
$$

The significance of the quantity $M$ relates to the design of cascaded multistage amplifiers for minimum overall noise figure, ${ }^{1}$ since, due to the influence of stage gain $\left(G_{a}\right)$, the minimum overall noise figure of a cascade of such stages is obtained when each stage is designed so as to minimize $M$, rather than $F$. The tradeoff between $F, G_{a}$ and $M$ is now a well-established principle of amplifier design and has been experimentally verified in numerous publications [3]-[6].

Manuscript received August 17, 2021; revised October 23, 2021; accepted October 30, 2021. (Corresponding author: Clive Poole.)

The authors are with the Department of Electronic and Electrical Engineering, University College London, London WC1E 6BT, U.K. (e-mail: c.poole@ucl.ac.uk; r.grammenos@ucl.ac.uk).

Color versions of one or more figures in this article are available at https://doi.org/10.1109/TMTT.2021.3129496.

Digital Object Identifier 10.1109/TMTT.2021.3129496

${ }^{1}$ According to the convention, we are here using "noise factor" to refer to the numerical quantity and "noise figure" to refer to that the same quantity expressed in $\mathrm{dB}$.
Poole and Paul's original article presented closed-form equations for the minimum value of noise measure, $M_{\min }$, obtainable for a given transistor and the associated optimum source termination, $\Gamma_{\mathrm{om}}[1]$. The original article contained a number of errors, which were later identified by Liu et al. [7] with some suggested corrections. A response to Liu et al. [7] acknowledged the typographical errors in some of the equations and also acknowledged that the value of $M_{\text {min }}$ presented in the original article was incorrect but stated that the source of this error was "not clear."

In order to establish the source of the error in the $M_{\min }$ value calculated in [1], the authors have set about deriving the relevant equations again from first principles. This has revealed that the original equation for $M_{\min }$ presented as (11) in [1] is incorrect. In this article, we present a new, correct equation for $M_{\min }$, as well as a rederivation of the equation for $\Gamma_{\mathrm{om}}$, and demonstrate their correctness by means of both numerical calculation and computer simulation. The results obtained using the new $M_{\min }$ equation are also verified by comparison with the value of $M_{\min }$ for the same device obtained independently by Gardner and Paul [8] using the entirely different characteristic noise matrix approaches.

Another aspect of the original article that we wish to highlight in this article relates to possible errors in the measured noise parameters of the NE71083 transistor used in the original example. After the original article was published, Pospieszalski [9] showed that the noise parameters of an intrinsic transistor chip must satisfy the following inequality:

$$
2 \geq \frac{4 N T_{o}}{T_{\min }} \geq 1
$$

where $T_{o}$ is the standard noise temperature $(290 \mathrm{~K}), T_{\min }$ is the minimum noise temperature (the noise temperature corresponding to $F_{\min }$ ), and $N$ is a parameter defined by Lange [10] as $N=R_{n} G_{\text {opt }}$ and commonly referred to as the Lange noise parameter or the Lange invariant [11]. The right-hand side of (2) is a reflection of the fact that the magnitude of the correlation coefficient, $\rho$, between the twoport internal equivalent noise voltage and equivalent noise current, as originally defined by Rothe and Dahlke [12], must always be less than or equal to unity.

Taking the measured noise parameters for the NE71083 GaAs metal-semiconductor field-effect transistor (MESFET) used in [1], we find that the value of $4 N T_{0} / T_{\min }$ is calculated as 3.71. Similarly, the measured noise parameters for the FHX04 HEMT device used in Gardner and Paul's 
article [8] give a value of $4 N T_{0} / T_{\min }=4.54$. In both cases, therefore, we see that the measured noise parameters violate the conditions of (2). The most likely explanation for this could be parasitic oscillations of the device under measurement terminations, giving erroneous values of $R_{n}$. Strong capacitive feedback between drain and gate caused by package parasitics in packaged devices may also cause the left-hand side of (2) to be violated. Since both the NE71083 and the FHX04 violate condition (2), we have supplemented these devices here by also considering two other devices which do satisfy condition (2).

Finally, there is an implicit assumption that all noise parameter measurements are being made at $T_{o}=290 \mathrm{~K}$. The definition of $F_{\min }$ depends on this assumption being correct, which gives rise to another potential source of error in devicemeasured noise parameters. Working with equations based on noise temperatures, rather than noise factors, would provide for greater precision, as the assumption of $T_{o}=290 \mathrm{~K}$ is then made explicit, but we have decided to maintain the use of noise factor equations for this article to maintain consistency with Poole and Paul's original article and allow easy comparison of the equations presented.

The authors consider it important that the correct equations are now published as the original article has been cited several times [4], [13]. The authors wish to ensure that the correct equation for $M_{\min }$ presented here is on record, and the original, incorrect equation presented in [1] is no longer used.

It is worth noting here that the question of the optimum source termination for minimum noise measure of a microwave transistor was also addressed by Pospieszalski [14], which considered the equivalent noise temperature of the intrinsic device noise sources, and presented alternative equations for the optimum source impedance in terms of these noise temperatures.

\section{Circles of Constant Noise Measure}

Our approach here will be the same as that used in [1], namely, by first deriving equations for circles of constant noise measure in the complex $\Gamma_{S}$ plane and then defining $\Gamma_{\text {om }}$ as the center of the constant $M$ circle having zero radius.

The relationship between noise factor $F$ and source reflection coefficient, $\Gamma_{S}$, for a linear noisy two-port device is given by the well-known formula [15]

$$
F=F_{\min }+4 r_{n} \frac{\left|\Gamma_{S}-\Gamma_{\text {on }}\right|^{2}}{\left|1+\Gamma_{\text {on }}\right|^{2}\left(1-\left|\Gamma_{S}\right|^{2}\right)}
$$

where

$F_{\min }=$ minimum obtainable noise factor for that device;

$\Gamma_{\mathrm{on}}=$ optimum source reflection coefficient (for $F_{\min }$ );

$r_{n}=$ normalized equivalent input noise resistance $\left(=R_{n} / Z_{o}\right)$.

In order to obtain an expression for noise measure in terms of $\Gamma_{S}$, we combine (3) for $F$ with one of the standard equations for $G_{a}$, such as [16]

$$
G_{a}=\frac{\left|S_{21}\right|^{2}\left(1-\left|\Gamma_{S}\right|^{2}\right)}{\left|1-S_{11} \Gamma_{S}\right|^{2}-\left|S_{22}-\Delta \Gamma_{S}\right|^{2}} .
$$

Substituting (4) and (3) into (1) gives us the following equation for $M$ as a function of $\Gamma_{S}$ [6]:

$$
\begin{aligned}
M= & \left(\frac{\left|S_{21}\right|^{2}}{\left|1+\Gamma_{\text {on }}\right|^{2}}\right) \\
& \cdot\left(\frac{\left|1+\Gamma_{\text {on }}\right|^{2}\left(1-\left|\Gamma_{S}\right|^{2}\right)\left(F_{\text {min }}-1\right)+4 r_{n}\left|\Gamma_{S}-\Gamma_{\text {on }}\right|^{2}}{\left|S_{21}\right|^{2}\left(1-\left|\Gamma_{S}\right|^{2}\right)-\left|1-S_{11} \Gamma_{S}\right|^{2}+\left|S_{22}-\Delta \Gamma_{S}\right|^{2}}\right) .
\end{aligned}
$$

Expanding (5) and collecting $\Gamma_{S}$ terms give us the following:

$$
\begin{aligned}
\left|\Gamma_{S}\right|^{2}[ & {\left[M\left|1+\Gamma_{\text {on }}\right|^{2}\left(|\Delta|^{2}-\left|S_{21}\right|^{2}-\left|S_{11}\right|^{2}\right)\right.} \\
& \left.+\left|S_{21}\right|^{2}\left(\left|1+\Gamma_{\text {on }}\right|^{2}\left(F_{\text {min }}-1\right)-4 r_{n}\right)\right] \\
& +\Gamma_{S}\left(M\left|1+\Gamma_{\text {on }}\right|^{2} C_{1}+4 r_{n}\left|S_{21}\right|^{2} \Gamma_{\text {on }}^{*}\right) \\
& +\Gamma_{S}^{*}\left(M\left|1+\Gamma_{\text {on }}\right|^{2} C_{1}^{*}+4 r_{n}\left|S_{21}\right|^{2} \Gamma_{\text {on }}\right) \\
= & \left|S_{21}\right|^{2}\left[\left|1+\Gamma_{\text {on }}\right|^{2}\left(F_{\text {min }}-1\right)+4 r_{n}\left|\Gamma_{\text {on }}\right|^{2}\right] \\
& \quad-M\left|1+\Gamma_{\text {on }}\right|^{2}\left(\left|S_{22}\right|^{2}+\left|S_{21}\right|^{2}-1\right)
\end{aligned}
$$

where we have employed the following standard constants:

$$
\begin{aligned}
C_{1} & =S_{11}-S_{22}^{*} \Delta \\
\Delta & =S_{11} S_{22}-S_{12} S_{21} .
\end{aligned}
$$

Equation (6) is in the form of an equation for a circle in the $\Gamma_{S}$ plane, i.e.,

$$
\left|\Gamma_{S}\right|^{2}+\left|C_{S m}\right|^{2}-\Gamma_{S}^{*} C_{S m}-\Gamma_{S} C_{S m}^{*}=\gamma_{S m}^{2}
$$

where

$C_{S m}=$ center of the constant $M$ circle in the $\Gamma_{S}$ plane;

$\gamma_{S m}=$ radius of the constant $M$ circle in the $\Gamma_{S}$ plane.

Rearranging (6) into the form of (7) reveals the center, $C_{S_{m}}$, and radius, $\gamma_{S_{m}}$, of the constant $M$ circle on the $\Gamma_{S}$ plane, shown as (9) and (10) in Fig. 1.

We can derive the value of the minimum noise measure by considering the constant $M$ circle of zero radius [1]. This means setting $\gamma_{S m}$ equal to zero in (10) and solving the resulting quadratic in $M$. This results in the following:

$$
\begin{aligned}
& M_{\min } \\
& =\frac{\left|S_{21}\right|^{2}}{\left(2 a^{2}\left(\left|C_{1}\right|^{2}+t v\right)\right)} \\
& \quad \times\left[\left(1 6 r _ { n } ^ { 2 } \left(v^{2}+t^{2}\left|\Gamma_{\text {on }}\right|^{4}+2 \operatorname{Re}\left(C_{1}^{2} \Gamma_{\text {on }}^{2}\right)\right.\right.\right. \\
& \quad+2\left(\left|C_{1}\right|^{2}\left|\Gamma_{\text {on }}\right|^{2}-2 \operatorname{Re}\left(C_{1} \Gamma_{\text {on }}\right)\left(v-t\left|\Gamma_{\text {on }}\right|^{2}\right)\right. \\
& \left.\left.\quad \quad-t v\left|\Gamma_{\text {on }}\right|^{2}\right)\right) \\
& \quad+8 a^{2} b r_{n}\left(t^{2}\left|\Gamma_{\text {on }}\right|^{2}-v^{2}+\left(t v+2\left|C_{1}\right|^{2}\right)\left(1-\left|\Gamma_{\text {on }}\right|^{2}\right)\right. \\
& \left.\quad+2 \operatorname{Re}\left(C_{1} \Gamma_{\text {on }}\right)(t+v)\right) \\
& \left.\quad+a^{4} b^{2}\left((v-t)^{2}-4\left|C_{1}\right|^{2}\right)\right)^{1 / 2} \\
& \\
& \left.\quad+\left(4 r_{n}\left(v-t\left|\Gamma_{\text {on }}\right|^{2}-2 \operatorname{Re}\left(C_{1} \Gamma_{\text {on }}\right)\right)-a^{2} b(t+v)\right)\right]
\end{aligned}
$$

where we have defined the following new constants:

$$
\begin{aligned}
a & =\left|1+\Gamma_{\text {on }}\right| \\
b & =\left(F_{\min }-1\right) \\
t & =\left(|\Delta|^{2}-\left|S_{21}\right|^{2}-\left|S_{11}\right|^{2}\right) \\
v & =\left(1-\left|S_{22}\right|^{2}-\left|S_{21}\right|^{2}\right) .
\end{aligned}
$$




$$
\begin{gathered}
C_{S m}=\frac{-M\left|1+\Gamma_{o n}\right|^{2} C_{1}^{*}-4 r_{n}\left|S_{21}\right|^{2} \Gamma_{o n}}{M\left|1+\Gamma_{o n}\right|^{2}\left(|\Delta|^{2}-\left|S_{21}\right|^{2}-\left|S_{11}\right|^{2}\right)+\left|S_{21}\right|^{2}\left(\left|1+\Gamma_{o n}\right|^{2}\left(F_{m i n}-1\right)-4 r_{n}\right)} \\
\gamma_{S m}=\sqrt{\frac{M\left|1+\Gamma_{o n}\right|^{2}\left(1-\left|S_{22}\right|^{2}-\left|S_{21}\right|^{2}\right)+\left|S_{21}\right|^{2}\left[\left|1+\Gamma_{o n}\right|^{2}\left(F_{\text {min }}-1\right)+4 r_{n}\left|\Gamma_{o n}\right|^{2}\right]}{M\left|1+\Gamma_{o n}\right|^{2}\left(|\Delta|^{2}-\left|S_{21}\right|^{2}-\left|S_{11}\right|^{2}\right)+\left|S_{21}\right|^{2}\left(\left|1+\Gamma_{o n}\right|^{2}\left(F_{\text {min }}-1\right)-4 r_{n}\right)}+\left|C_{S_{m}}\right|^{2}} \\
\Gamma_{o m}=\frac{-M_{\text {min }}\left|1+\Gamma_{o n}\right|^{2} C_{1}^{*}-4 r_{n}\left|S_{21}\right|^{2} \Gamma_{o n}}{M_{\text {min }}\left|1+\Gamma_{o n}\right|^{2}\left(|\Delta|^{2}-\left|S_{21}\right|^{2}-\left|S_{11}\right|^{2}\right)+\left|S_{21}\right|^{2}\left(\left|1+\Gamma_{o n}\right|^{2}\left(F_{\text {min }}-1\right)-4 r_{n}\right)}
\end{gathered}
$$

Fig. 1. New equations for center and radius of a constant $M$ circle and $\Gamma_{\mathrm{om}}$.

With the value of $M_{\min }$ determined by (8), we can determine the associated source reflection coefficient, $\Gamma_{\mathrm{om}}$, as being the center of the $M_{\min }$ noise measure circle. Substituting $M_{\min }$ into (9) results in the equation for $\Gamma_{\text {om }}$ given as (11) in Fig. 1.

\section{VERIFICATION}

We will now repeat the design example used in Poole and Paul's original article [1] but now using the newly derived equations. The design example employed an NE71083 GaAs MESFET at a center frequency of $10 \mathrm{GHz}$ and bias conditions $V_{d s}=3.0 \mathrm{~V}$ and $l_{d}=8 \mathrm{~mA}$. The measured $S$-parameters of the transistor in the common-source configuration were given in [1] as

$$
\left[\begin{array}{ll}
S_{11} & S_{12} \\
S_{21} & S_{22}
\end{array}\right]=\left[\begin{array}{cc}
0.724 \angle 46^{\circ} & 0.716 \angle-47^{\circ} \\
1.303 \angle-106^{\circ} & 0.616 \angle 64^{\circ}
\end{array}\right]
$$

The measured noise parameters at the same bias conditions are $F_{\min }=1.7 \mathrm{~dB}, \Gamma_{\text {on }}=0.620 \angle 148^{\circ}$, and $R_{n}=12 \Omega$.

Using (8), the minimum noise measure obtainable with this device is calculated as $M_{\min }=1.006$, and using (11), the source reflection coefficient, $\Gamma_{\mathrm{om}}$, required to achieve this is calculated as $0.560 \angle 161.0^{\circ}$. These values differ from that reported in Poole and Paul's original article [1], with $M_{\min }$ being calculated here as 1.006 rather than 1.303. For a cascade of multiple stages all having the same noise measure, $M$, the overall noise factor of the cascade (assuming a large enough stage gain) can be approximated by

$$
F_{\text {cascade }}=M+1 .
$$

It follows, therefore, that the cascade having the minimum overall noise factor will be the cascade of $M_{\min }$ stages. The consequence of the error in the value of $M_{\min }$ outlined above, for a cascade of such identical stages, is that the noise factor of the cascade would be calculated as 2.303 (or $3.6 \mathrm{~dB}$ ) using (11) from [1], rather than the correct value of 2.006 (or $3.0 \mathrm{~dB}$ ) using (8), here an error of around $0.6 \mathrm{~dB}$. The value of $\Gamma_{\mathrm{om}}$, however, is close to that calculated in Poole and Paul's original article since (11) in Fig. 1 is essentially the same as (12) in the original article [1]. The difference in calculated $\Gamma_{\text {om }}$ is small enough to be attributed to a rounding error (MATLAB was used to calculate $\Gamma_{\mathrm{om}}=0.560 \angle 161.0^{\circ}$ for this article, whereas the value $\Gamma_{\mathrm{om}}=0.554 \angle 161.0^{\circ}$ presented in the original article was calculated manually), whereas the difference in the value of $M_{\min }$ suggests that (11) in Poole and Paul's original article may, in fact, be incorrect. We will now proceed to demonstrate below the correctness of the new (8), and 1.006 is, indeed, the correct value of $M_{\min }$ for this device.

First, the $M_{\min }$ value can be verified by calculating $F$ and $G_{a}$ directly by setting the source $\Gamma_{S}$ equal to $\Gamma_{\mathrm{om}}$ in (3) and (4), respectively, and applying the $F$ and $G_{a}$ values, thus obtained (1). With $\Gamma_{S}$ set to $0.560 \angle 161.0^{\circ}$, (3) yields $F=1.569(2.0 \mathrm{~dB})$, and (4) yields $G_{a}=2.302(3.6 \mathrm{~dB})$. Equation (1) then gives us an $M$ value of 1.006, as expected.

Whether or not we can match the input port of the device with the $\Gamma_{\mathrm{om}}$ to realize a minimum noise measure amplifier depends on where $\Gamma_{\mathrm{om}}$ lies in relation to any regions of instability in the source plane. We can establish the stability of the device using the Wood/Rollet criteria [17].

The values of $K$ and $|\Delta|$ quoted in [1] have been verified as $K=0.681$ and $|\Delta|=1.080$, meaning that the device is potentially unstable. The center and radius of the source plane stability circle are determined as follows, using standard formulae for these quantities [18]:

$$
\begin{aligned}
C_{\mathrm{SS}} & =\frac{C_{1}^{*}}{\left|S_{11}\right|^{2}-|\Delta|^{2}}=1.060 \angle 78^{\circ} \\
r_{\mathrm{SS}} & =\frac{\left|S_{12} S_{21}\right|}{\left.|| S_{11}\right|^{2}-|\Delta|^{2} \mid}=1.443 .
\end{aligned}
$$

The source plane stability circle together with $\Gamma_{\mathrm{on}}, \Gamma_{\mathrm{om}}$, and various circles of constant noise measure on the source reflection coefficient plane are shown in Fig. 2, which is similar to Fig. 3 of [1]. The circles of constant noise measure, in this case, have been determined using (9) and (10) above.

Since the stability circle in Fig. 2 encompasses the origin, the stable region is represented by the interior of the stability circle. Both $\Gamma_{\text {on }}$ and $\Gamma_{\text {om }}$ lie inside the stable region in the source reflection coefficient plane, so it is theoretically possible to build a stable amplifier having the minimum noise measure of 1.006 with this device.

The noise measure calculated for the same device with input matched for minimum noise figure (i.e., $\Gamma_{S}=\Gamma_{\text {on }}$ ) is here calculated as 1.291 (which differs from the 1.353 value quoted in [1]). By plotting the $M=1.291$ noise measure circle in Fig. 2, we can confirm that $\Gamma_{\text {on }}$ does, in fact, lie on this circle.

We applied the PathWave advanced design system (ADS) software package from Keysight [19] to verify the correctness of (8) and (11) by determining the available gain and noise 


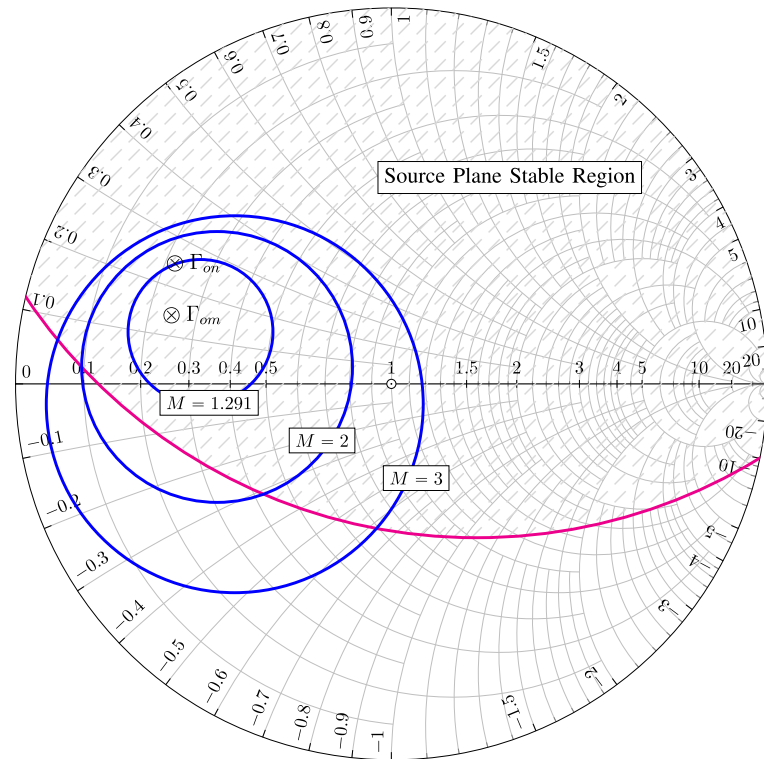

Fig. 2. Source plane constant noise measure circles, stability circle, $\Gamma_{\text {on }}$, and $\Gamma_{\mathrm{om}}$ for $\mathrm{NE} 71083$ at $10 \mathrm{GHz}\left(V_{d s}=3.0 \mathrm{~V}\right.$ and $\left.l_{d}=8 \mathrm{~mA}\right)$.

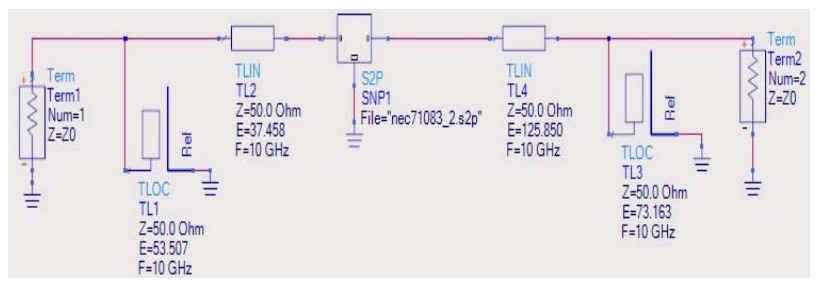

Fig. 3. ADS test circuit.

figure of a single-stage NE71083 amplifier with various values of source termination.

The test circuit used in ADS is shown in Fig. 3. A single stub input matching network was used to present the required value of $\Gamma_{S}$ to the input of the transistor. Different values of $\Gamma_{S}$ were then implemented by changing the electrical lengths of the input transmission line and the open circuit stub. Another single stub matching network at the output port was used to conjugately match the corresponding output impedance of the transistor to the load $(50 \Omega)$ for any given value of $\Gamma_{S}$. The specific line and stub lengths shown in the example circuit of Fig. 3 are those for the minimum noise measure circuit (i.e., $\Gamma_{S}=\Gamma_{\text {om }}$ ).

The values of $F$ and $G_{a}$ obtained from the ADS circuit in Fig. 3 were then applied to (1) to calculate the value of $M$ associated with that source termination. The corresponding value of $\Gamma_{\text {out }}$ given by ADS was used to design the output matching network for the conjugate match at the output port. The source reflection coefficients chosen represented a few points that all lying on one of the three constant noise measure circles shown in Fig. 2, spaced at random intervals around the circumference of each circle.

We have restricted our analysis to only values of $\Gamma_{S}$ that lie inside the source plane stable region in Fig. 2. Table I shows the values of $\Gamma_{S}$ used for this exercise, together with the corresponding value of $\Gamma_{\text {out }}, G_{a}$, and $F$ given by ADS. The columns labeled TL1-TL4 contain the design values of electrical length for the input lines and stubs, as defined in Fig. 3, in degrees. The design parameters and results for the two optimum source terminations, $\Gamma_{\text {om }}$ and $\Gamma_{\text {on }}$, are also included in Table I for reference.

From Table I, we can see that, with the various test values of $\Gamma_{S}$ chosen, the values of noise measure provided by ADS correspond to those that we would expect, according to the constant $M$ circle on which the $\Gamma_{S}$ point is located in Fig. 2. We consider this to be strong verification that (8)-(11) are giving correct results.

As further verification of the equations derived in this article, we can also use (8) to calculate $M_{\min }$ for the FHX04 HEMT device from Sumitomo Electric, which was used in Gardner and Paul's 1997 article on optimum noise measure [8], and compare our result with theirs. The device data given in [8] are as given follows:

$$
\left[\begin{array}{ll}
S_{11} & S_{12} \\
S_{21} & S_{22}
\end{array}\right]=\left[\begin{array}{cc}
0.653 \angle-159.8^{\circ} & 0.076 \angle-4^{\circ} \\
2.512 \angle 20.2^{\circ} & 0.552 \angle-125.7^{\circ}
\end{array}\right] .
$$

The noise parameters at the same bias conditions are $F_{\min }=$ $0.66 \mathrm{~dB}, \Gamma_{\text {on }}=0.52 \angle 134^{\circ}$, and $R_{n}=7 \Omega$.

Applying these data to (8), we obtain the same value of $M_{\min }=0.177$ that Gardner and Paul calculated [8]. It is noteworthy that the value of $M_{\min }$ calculated by Gardner and Paul in their article is arrived at using an entirely different characteristic noise matrix methodology.

\section{Sensitivity Analysis}

This brings us back to consideration of the impact of possible errors in the measured noise parameters alluded to earlier. We conducted a preliminary investigation of the sensitivity of $M_{\min }$, calculated by (8), to variations in the four noise parameters of the device $\left(F_{\min }, R_{n},\left|\Gamma_{\text {on }}\right|\right.$, and $\left.\angle \Gamma_{\text {on }}\right)$ for four sample devices: the two devices used in Poole and Paul's, and Paul and Gardner's original articles, plus two other devices that we know satisfy Pospieszaki's inequality (2). Fig. 4 shows percentage change in $M_{\text {min }}$ with percentage change in each of the four noise parameters (with the other three held constant) for the NE71083 device used in [1].

We can observe that $M_{\min }$ is highly sensitive to the value of $\Gamma_{\text {on. }}$ Fig. 4 shows that a $20 \%$ error in the value of $\angle \Gamma_{\text {on }}$, for example, could result in an error of up to $40 \%$ in the calculated value of $M_{\min }$ for this device. Translating into overall noise figure of a cascade of $M_{\text {min }}$ stages means that a $20 \%$ error in the value of $\angle \Gamma_{\text {on }}$ used would result in the overall noise figure of the cascade being out by almost $1 \mathrm{~dB}$ [according to (12)]. $M_{\text {min }}$ was less sensitive to errors in the other device noise parameters, with the least sensitivity being shown to errors in $R_{n}$. These sensitivities will vary from device to device, and considering (1), it is reasonable to expect that devices with higher gain will exhibit lower sensitivity of $M_{\min }$ to errors in device noise parameters.

For comparison, we also examined the sensitivity of two devices that satisfy Pospieszaki's inequality (2). We chose to use two of the devices referenced by Pospieszaki in his article [9], namely, the Avago ATF-34143 HEMT and the 
TABLE I

ADS Test Configuration AND Results For NE71083 With VARious VALues of $\Gamma_{S}$

Optimum Source Terminations

\begin{tabular}{cccccccc}
\hline \hline$\Gamma_{S}$ & TL1 & TL2 & $\Gamma_{\text {out }}$ & TL3 & TL4 & Ga & F \\
\hline$\Gamma_{\text {om }}=0.560 \angle 161.0^{\circ}$ & $53.507^{\circ}$ & $37.458^{\circ}$ & $0.856 \angle 40.5^{\circ}$ & $73.163^{\circ}$ & $125.85^{\circ}$ & 2.304 & 1.569 \\
$\Gamma_{\text {on }}=0.620 \angle 148.0^{\circ}$ & $57.677^{\circ}$ & $41.842^{\circ}$ & $0.826 \angle 36.2^{\circ}$ & $71.186^{\circ}$ & $125.256^{\circ}$ & 1.590 & 1.479
\end{tabular}

$M=1.291$ Constant $M$ Circle.

\begin{tabular}{cccccccc}
\hline \hline$\Gamma_{S}$ & TL1 & TL2 & $\Gamma_{\text {out }}$ & TL3 & TL4 & Ga & F \\
\hline $0.718 \angle 166.5^{\circ}$ & $64.140^{\circ}$ & $28.805^{\circ}$ & $0.926 \angle 38.3^{\circ}$ & $78.469^{\circ}$ & $120.242^{\circ}$ & 2.684 & 1.810 \\
$0.375 \angle 149.5^{\circ}$ & $38.974^{\circ}$ & $49.238^{\circ}$ & $0.754 \angle 43.7^{\circ}$ & $66.454^{\circ}$ & $132.391^{\circ}$ & 2.116 & 1.681 \\
$0.461 \angle-173.9^{\circ}$ & $46.095^{\circ}$ & $28.224^{\circ}$ & $0.902 \angle 48.7^{\circ}$ & $76.559^{\circ}$ & $127.099^{\circ}$ & 4.725 & 2.018 \\
$0.704 \angle 157.9^{\circ}$ & $63.232^{\circ}$ & $33.661^{\circ}$ & $0.888 \angle 36.6^{\circ}$ & $75.503^{\circ}$ & $121.976^{\circ}$ & 1.852 & 1.594 \\
$0.359 \angle 177.5^{\circ}$ & $37.571^{\circ}$ & $35.731^{\circ}$ & $0.829 \angle 49.5^{\circ}$ & $71.333^{\circ}$ & $131.78^{\circ}$ & 3.266 & 1.896 \\
$0.643 \angle 180.0^{\circ}$ & $59.250^{\circ}$ & $24.978^{\circ}$ & $0.955 \angle 43.0^{\circ}$ & $81.175^{\circ}$ & $120.112^{\circ}$ & 6.070 & 2.078
\end{tabular}

$M=2.000$ Constant $M$ Circle.

\begin{tabular}{cccccccc}
\hline \hline$\Gamma_{S}$ & TL1 & TL2 & $\Gamma_{\text {out }}$ & TL3 & TL4 & Ga & F \\
\hline $0.613 \angle 138.8^{\circ}$ & $57.201^{\circ}$ & $46.682^{\circ}$ & $0.79 \angle 34.4^{\circ}$ & $68.800^{\circ}$ & $126.115^{\circ}$ & 1.355 & 1.524 \\
$0.826 \angle 174.5^{\circ}$ & $71.127^{\circ}$ & $19.907^{\circ}$ & $0.99 \angle 38.2^{\circ}$ & $85.996^{\circ}$ & $113.082^{\circ}$ & 12.422 & 2.839 \\
$0.123 \angle 148.1^{\circ}$ & $13.943^{\circ}$ & $57.422^{\circ}$ & $0.66 \angle 55.3^{\circ}$ & $60.327^{\circ}$ & $142.031^{\circ}$ & 2.506 & 2.202 \\
$0.778 \angle 157.5^{\circ}$ & $68.010^{\circ}$ & $30.701^{\circ}$ & $0.907 \angle 35.1^{\circ}$ & $76.944^{\circ}$ & $119.981^{\circ}$ & 1.611 & 1.759 \\
$0.271 \angle-135^{\circ}$ & $29.383^{\circ}$ & $14.638^{\circ}$ & $0.865 \angle 63.1^{\circ}$ & $73.801^{\circ}$ & $136.627^{\circ}$ & 6.042 & 2.669 \\
$0.415 \angle 126.8^{\circ}$ & $42.360^{\circ}$ & $59.343^{\circ}$ & $0.691 \angle 38.5^{\circ}$ & $62.397^{\circ}$ & $132.371^{\circ}$ & 1.596 & 1.747
\end{tabular}

$M=3.000$ Constant $M$ Circle.

\begin{tabular}{cccccccc}
\hline \hline$\Gamma_{S}$ & TL1 & TL2 & $\Gamma_{\text {out }}$ & TL3 & TL4 & Ga & F \\
\hline $0.855 \angle 163.6^{\circ}$ & $73.128^{\circ}$ & $23.815^{\circ}$ & $0.952 \angle 35.1^{\circ}$ & $80.882^{\circ}$ & $116.468^{\circ}$ & 1.987 & 2.491 \\
$0.09 \angle-21.8^{\circ}$ & $10.245^{\circ}$ & $143.318^{\circ}$ & $0.573 \angle 71.5^{\circ}$ & $54.399^{\circ}$ & $153.298^{\circ}$ & 2.830 & 2.940 \\
$0.214 \angle-73.4^{\circ}$ & $23.661^{\circ}$ & $165.537^{\circ}$ & $0.744 \angle 80.3^{\circ}$ & $65.817^{\circ}$ & $151.114^{\circ}$ & 4.845 & 3.381 \\
$0.105 \angle-36.9^{\circ}$ & $11.924^{\circ}$ & $150.443^{\circ}$ & $0.594 \angle 73.8^{\circ}$ & $55.899^{\circ}$ & $153.690^{\circ}$ & 3.033 & 3.011 \\
$0.112 \angle 56.7^{\circ}$ & $12.681^{\circ}$ & $103.455^{\circ}$ & $0.524 \angle 59.4^{\circ}$ & $50.928^{\circ}$ & $148.904^{\circ}$ & 2.219 & 2.648 \\
$0.281 \angle 98.7^{\circ}$ & $30.353^{\circ}$ & $77.480^{\circ}$ & $0.569 \angle 42.7^{\circ}$ & $54.157^{\circ}$ & $139.011^{\circ}$ & 1.684 & 2.218
\end{tabular}

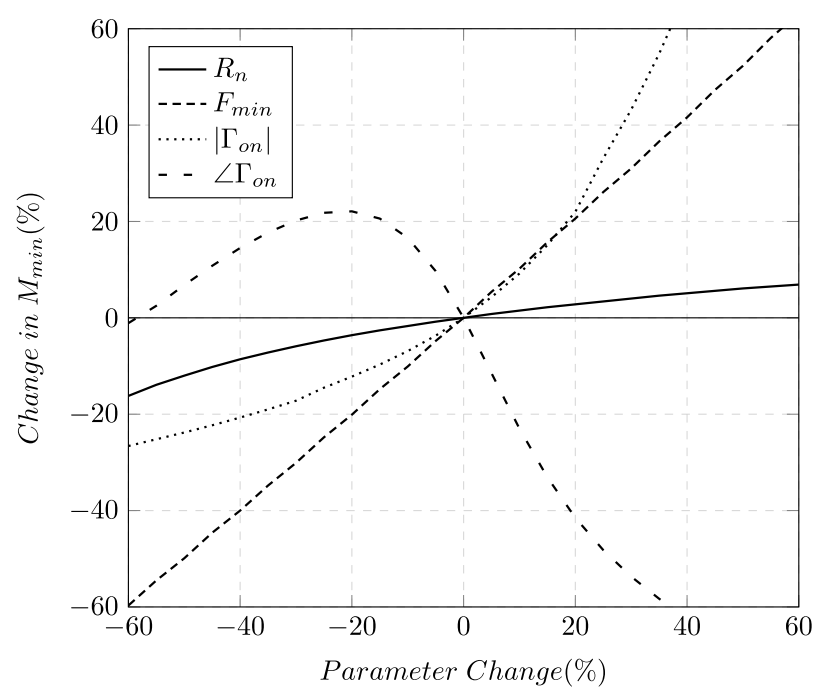

Fig. 4. Sensitivity of $M_{\min }$ to the four noise parameters of the NE71083 device used in [1].

Sumitomo FHX45X HEMT. Typical $S$-parameters and noise parameters for these devices at $10 \mathrm{GHz}$ were obtained from the manufacturer's data sheets with the bias conditions for the
TABLE II

$M_{\text {Min }}$ Sensitivity to Device Noise Parameter ERror

\begin{tabular}{lcccccc}
\hline \multirow{2}{*}{ Device } & $\left(\frac{4 N T_{o}}{T_{\min }}\right)$ & $G_{a}$ & \multicolumn{5}{c}{ Sensitivity } \\
\cline { 4 - 7 } NE71083 & 4.54 & $3.6 d B$ & $76 \%$ & $16 \%$ & $130 \%$ & $76 \%$ \\
FHX04 & 3.71 & $11.3 d B$ & $69 \%$ & $<1 \%$ & $4 \%$ & $15 \%$ \\
FHX45X & 1.79 & $13.1 d B$ & $65 \%$ & $<1 \%$ & $13 \%$ & $8 \%$ \\
ATF-34143 & 1.67 & $7.1 d B$ & $74 \%$ & $<1 \%$ & $10 \%$ & $8 \%$ \\
\hline
\end{tabular}

ATF-34143 being $V_{\mathrm{DS}}=3 \mathrm{~V}$ and $I_{\mathrm{DS}}=20 \mathrm{~mA}$ and those for the FHX45X being $V_{\mathrm{DS}}=2 \mathrm{~V}$ and $I_{\mathrm{DS}}=10 \mathrm{~mA}$. Under these conditions, the calculated value of $4 N T_{0} / T_{\min }$ for both devices is less than 2, being 1.67 for the ATF-34143 and 1.79 for the FHX45X. Applying (8), the calculated values of $M_{\min }$ are 0.382 , and 0.124 , respectively.

Table II shows the sensitivity of $M_{\min }$ with each of the four noise parameters varied independently over a range of $\pm 60 \%$ for all four devices. 
Comparing the data in Table II, we can see that, with the exception of sensitivity to errors in $F_{\min }$, which was approximately linear with the near-unity slope in all cases, the sensitivity of $M_{\min }$ to errors in the noise parameters was far higher in the case of the NE71083 than for the other three devices, where errors in these noise parameters of up to $\pm 60 \%$ resulted in no more than a $15 \%$ variation in the calculated value of $M_{\mathrm{min}}$. In the case of errors in $R_{n}$, this variation is less than $1 \%$. Although Table II appears to indicate a trend toward lower sensitivity for smaller values of $4 N T_{0} / T_{\min }$, the most significant factor appears to be the available power gain of the device, $G_{a}$, under minimum noise measure conditions (i.e., $\Gamma_{S}=\Gamma_{\text {om }}$ ). The available power gain of the NE71083 is less than half that of any of the other devices under the same matching conditions, which does suggest a link between device available gain and sensitivity of $M_{\min }$ to errors in the measured noise parameters that may be worthy of further research.

\section{CONCLusion}

Correct equations are presented here for circles of constant noise measure, minimum noise measure $\left(M_{\min }\right)$, and the associated source termination $\left(\Gamma_{\mathrm{om}}\right)$. These may be used to design individual stages in a cascaded multistage low-noise amplifier to achieve a minimum overall noise figure. The new equations replace those previously published by one of the authors and have been verified as correct by numerical calculation, computer simulation, and comparison with results obtained by other authors using an entirely different method. This article confirms that the original equation for minimum noise measure, $M_{\min }$, [1, eq. (11)] gives incorrect results and should, therefore, no longer be used. Equation (8) published here should be used in its place.

\section{ACKNOWLEDGMENT}

This article is presented for publication in memory of Dr. D. K. Paul who coauthored the original article on Minimum Noise Measure and who passed away earlier this year. The authors would like to thank Prof. C. P. Liu and Prof. I. Z. Darwazeh, both of UCL, for their invaluable help and advice.

\section{REFERENCES}

[1] C. R. Poole and D. K. Paul, "Optimum noise measure terminations for microwave transistor amplifiers (short paper)," IEEE Trans. Microw. Theory Techn., vol. MTT-33, no. 11, pp. 1254-1257, Nov. 1985.

[2] H. A. Haus and R. B. Adler, "Optimum noise performance of linear amplifiers," Proc. IRE, vol. 46, no. 8, pp. 1517-1533, Aug. 1958.

[3] J. Engberg and G. Gawler, "Significance of the noise measure for cascaded stages," IEEE Trans. Circuit Theory, vol. CT-16, no. 2, pp. 259-260, May 1969.

[4] A. Victor and J. Nath, "An analytic technique for trade-off of noise measure and mismatch loss for low noise amplifier design," in Proc. IEEE Int. Conf. Wireless Microw. Technol. (WAMICON), Apr. 2010, pp. $1-4$.

[5] A. Huber, C. Bergamaschi, R. Bauknecht, H. Jackel, and H. Melchior, "Minimization of the noise measure of InP/InGaAs HBTs," in Proc. Int. Conf. Indium Phosph. Related Mater., May 1997, pp. 649-652.

[6] C. Poole and I. Darwazeh, Microwave Active Circuit Analysis and Design. Amsterdam, The Netherlands: Elsevier, 2015.
[7] J. C. Liu, S. S. Bor, P. C. Lu, D. Paul, P. Gardner, and C. Poole, "Comments, with reply, on 'Optimum noise measure terminations for microwave transistor amplifiers' by C.R. Poole and D.K. Paul,' IEEE Trans. Microw. Theory Techn., vol. 41, no. 2, pp. 363-364, Feb. 1993.

[8] P. Gardner and D. K. Paul, "Optimum noise measure configurations for transistor negative resistance amplifiers," IEEE Trans. Microw. Theory Techn., vol. 45, no. 5, pp. 580-586, May 1997.

[9] M. W. Pospieszalski, "Interpreting transistor noise," IEEE Microw. Mag., vol. 11 , no. 6, pp. 61-69, Oct. 2010.

[10] J. Lange, "Noise characterization of linear twoports in terms of invariant parameters," IEEE J. Solid-State Circuits, vol. JSSC-2, no. 2, pp. 37-40, Jun. 1967.

[11] L. Boglione, "An original demonstration of the $t_{\min } / t_{o} \leq 4 N$ inequality for noisy two-port networks," IEEE Trans. Microw. Wireless Compon. Lett., vol. 18, no. 5, pp. 326-328, May 2008.

[12] H. Rothe and W. Dahlke, "Theory of noisy fourpoles," Proc. IRE, vol. 44, no. 6, pp. 811-818, Jun. 1956.

[13] R. B. Hallgren, "A matrix formulation for noise transduction as a general case of noise measure," IEEE Microw. Guided Wave Lett., vol. 2, no. 1, pp. 14-16, Jan. 1992.

[14] M. W. Pospieszalski, "Modeling of noise parameters of MESFETs and MODFETs and their frequency and temperature dependence," IEEE Trans. Microw. Theory Techn., vol. 37, no. 9, pp. 1340-1350, Sep. 1989.

[15] R. P. Meys, "A wave approach to the noise properties of linear microwave devices," IEEE Trans. Microw. Theory Techn., vol. MTT-26, no. 1, pp. 34-37, Jan. 1978.

[16] G. Gonzalez, Microwave Transistor Amplifiers, Analysis and Design, 2nd ed. Englewood Cliffs, NJ, USA: Prentice-Hall, 1997.

[17] D. Woods, "Reappraisal of the unconditional stability criteria for active 2-port networks in terms of S parameters," IEEE Trans. Circuits Syst., vol. CAS-23, no. 2, pp. 73-81, Feb. 1976.

[18] G. Bodway, "Two-port power flow analysis using generalised scattering parameters," Microw. J., vol. 10, pp. 61-69, May 1967.

[19] Keysight Technologies. (2021) Pathwave Advanced Design System $(A D S)$. [Online]. Available: https://www.keysight.com/zz/en/products/ software/pathwave-design-software/pathwave-advanced-designsystem.html

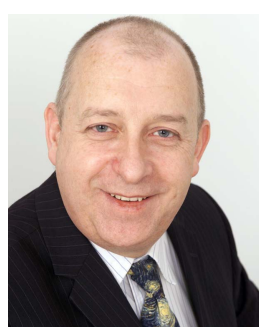

Clive Poole worked in the global electronics and telecommunications industries, as well as academia, for nearly 40 years, having held senior positions in various design houses, manufacturing organizations, and telecommunications operating companies. He is currently an Associate Professor (Teaching) with the Department of Electronic and Electrical Engineering, University College London (UCL), London, U.K. His teaching and research interests include $\mathrm{RF}$ and microwave circuit design, mobile communications systems, and telecommunications business strategy and finance.

Prof. Poole is also a Chartered Engineer (CEng) and a Fellow of the Institution of Engineering and Technology (FIET).

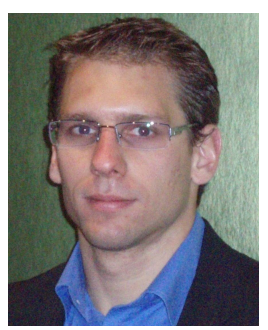

Ryan Grammenos (Member, IEEE) is currently an Associate Professor (Teaching) with the Department of Electronic and Electrical Engineering, University College London (UCL), London, U.K. His research interests include signal processing for communications, software-defined radio, and the Internet of Things. He has led or co-led numerous research and education projects, including the development of a proof-of-concept, precommercialization $5 \mathrm{G}$ transceiver prototype in collaboration with the industry. $\mathrm{He}$ also leads and teaches a number of courses in telecommunications and connected systems.

Prof. Grammenos is also a member of IET and a Senior Fellow of the Higher Education Academy (SFHEA). 